\title{
Multisite study of Titan SGS stapler in longitudinal gastric resection
}

\author{
Christen E. Salyer ${ }^{1}$ (D) Jonathan Thompson ${ }^{3}$. Aaron Hoffman ${ }^{4} \cdot$ Matthew D. Burstein $^{4} \cdot$ Paul Enochs $^{5}$. \\ Brad M. Watkins ${ }^{2} \cdot$ Joshua Kuethe ${ }^{2} \cdot$ Michael D. Goodman $^{1,6}$
}

Received: 23 June 2021 / Accepted: 11 January 2022 / Published online: 26 January 2022

(C) The Author(s), under exclusive licence to Springer Science+Business Media, LLC, part of Springer Nature 2022

\begin{abstract}
Background Standardization of the laparoscopic sleeve gastrectomy procedure is needed to improve patient outcomes. A single-fire $23 \mathrm{~cm}$ stapler was developed to streamline the operation. Comparative testing conducted on excised human tissue has demonstrated the superiority of the novel Titan SGS stapler to two commonly utilized commercial devices in both staple line integrity and burst pressure. We hypothesized that the stapler would be safe and effective in creating longitudinal gastric resections in human patients.

Methods 61 patients were enrolled to undergo gastric resection with the Titan SGS stapler. Perioperative interventions and post-operative adverse events were recorded. Upper GI study was completed on post-operative day 1, and patients were followed for 6 weeks post-operatively to determine any subacute device-related adverse events.

Results Surgeon feedback for intraoperative device utilization and post-operative gastric pouch shape were positive. Adverse events were found to be mild, limited, and generally well-known effects of bariatric surgery. One episode of post-operative hemorrhage required surgical takeback, with no criminal bleeding vessel identified.
\end{abstract}

Conclusion The Titan SGS stapler is both safe and effective in sleeve gastrectomy pouch creation.

Keywords Stomach stapling $\cdot$ Surgical staples $\cdot$ Sleeve gastrectomy

As the prevalence of obesity continues to surge in the United States, with rates in the adult population greater than $42 \%$ in 2018 [1], laparoscopic sleeve gastrectomy (LSG) has become the most common bariatric procedure performed, accounting for $61.4 \%$ of all bariatric procedures completed in 2018 [2]. Numerous studies have confirmed both the efficacy and safety of this procedure [3-6]. However, differences

Michael D. Goodman

michael.goodman@uc.edu

1 Division of Research, Section of General Surgery, Department of Surgery, University of Cincinnati, Cincinnati, $\mathrm{OH}, \mathrm{USA}$

2 Section of General Surgery, University of Cincinnati Medical Center, Cincinnati, OH, USA

3 Standard Bariatrics, Inc., Cincinnati, OH, USA

4 Section of General Surgery, State University of New York at Buffalo, Buffalo, NY, USA

5 WakeMed, Cary, NC, USA

6 Division of Research, Department of Surgery, University of Cincinnati College of Medicine, 231 Albert Sabin Way (ML 0558), Cincinnati, OH 45267-0558, USA in operative techniques such as oversewing, bougie size, and distance of the staple line from the pylorus may lead to less than optimal outcomes [7]. Ideal tubular sleeve anatomy is achieved in less than $40 \%$ of radiologically studied sleeves resulting in variable outcomes for the patients, including reduced weight loss efficiency and gastroesophageal reflux [8]. Recent efforts have been made towards standardization of the procedure in order to improve patient outcomes, decrease operative time, and reduce costs $[9,10]$.

The Titan SGS stapler is a novel stapling device that was developed to optimize and streamline the LSG operation, utilizing a single-fire staple mechanism instead of at least three staple loads required to traverse the length of the gastric greater curvature. The proposed benefits of this stapler include decreased operative time, removal of junctions in the staple line, and elimination of angulation between staple loads. Without multiple crossed staple lines, the Titan eliminates the surgeons' search for and removal of the migratory "crotch staple" which has been identified as a risk of leak [11]. Comparative testing conducted on excised human tissue revealed the superiority of the Titan SGS stapler to two commonly utilized commercial devices in both staple 
line integrity and burst pressure, noting that over one third of experimentally created leaks occur at stapler junctions [12]. The Titan SGS has additionally been demonstrated as safe and effective in gastric resection in acute and chronic porcine studies.

This study is the first experience for use of the Titan SGS stapler in humans. The purpose of this study was to demonstrate the safety and usability of the Titan SGS stapler in creating longitudinal gastric resections, with safety defined by the absence of device-related adverse events in the study period and usability evaluated based on surgeon impression of the device and resulting gastric pouch following each use. We hypothesized that use of the Titan SGS stapler would be both safe and effective in longitudinal gastric resection.

\section{Materials and methods}

This was a multisite, open-label study of the Titan SGS stapler for use in longitudinal gastric resection. The study protocol was approved by the Institutional Review Board at Advarra (Pro00041969) with reliance reviews performed at each clinical site. The investigational device study was conducted under a Food and Drug Administration (FDA) investigational device exemption (IDE) G200085. The trial was carried out in accordance with International Conference on Harmonization Good Clinical Practice (ICH GCP) and the United States (US) Code of Federal Regulations (CFR) applicable to clinical studies (45 CFR Part 46, 21 CFR Part 50, 21 CFR Part 56 and 21 CFR Part 812) and was registered on clinicalTrials.gov (NCT04347837).

\section{Titan SGS stapler}

The Titan SGS is a single-use sterile stapler used for stapling and cutting during laparoscopic and open surgical procedures. This novel stapler is designed for single-staple load creation of a longitudinal gastric resection line. The device forms staples in the standard "B" shape to secure targeted tissue that uniquely (compared to current commercial staplers) range in closed staple height of 1.2 to $2.2 \mathrm{~mm}$ along the single-staple line. The stapling device along with its cable and power supply unit are seen in Fig. 1. A representative image of the stapler traversing the gastric curve, prior to, and after firing staples is shown in Fig. 2. It is used with the Standard trocar, a $19 \mathrm{~mm}$ trocar which is adaptable for use with standard laparoscopic instruments, shown in Fig. 3.

\section{Patients}

Participants were selected from the trial physicians' existing patient populations and screened for eligibility. Inclusion criteria were defined as patients age 18 to 65 undergoing a

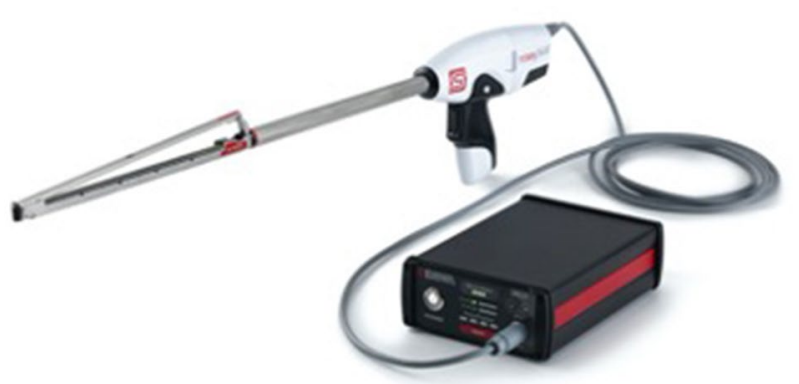

Fig. 1 Titan SGS stapling device and power cord

procedure requiring longitudinal gastric resection including laparoscopic sleeve gastrectomy, laparoscopic gastric wedge resection, and laparoscopic duodenal switch. Exclusion criteria included incarceration, prior gastric or foregut surgery, pre-existing bleeding disorder, systemic anticoagulation, and significant organ system disease (Stage III chronic kidney disease, liver cirrhosis, congestive heart failure with ejection fraction $<50 \%$, COPD with oxygen requirement, or uncontrolled diabetes mellitus as defined by hemoglobin A1c $>10)$. Qualifying patients were enrolled between July 2, 2020 and November 3, 2020 and underwent informed consent and documentation with trained study personnel.

\section{Endpoints}

The primary endpoint of this study was the rate of DeviceRelated Adverse Events (DRAEs) with a defined performance goal of 2 or fewer events, with secondary endpoints being the rate of Unanticipated Device-Related Adverse Effects (UADEs) by seriousness, as well as the rate of anticipated non-serious events, and the usability of Titan SGS in longitudinal gastric resection. DRAEs were defined as follows:

- Post-operative leak as defined by positive intraoperative air bubble leak test, post-operative upper GI or CT evaluation by radiologist, or re-operative findings consistent with gastric leak

- Abdominal/deep space abscess

- Staple line bleeding requiring transfusion or re-operation either intraoperatively or post-operatively within the first $72 \mathrm{~h}$ of surgery start time

- Gastric stricture as defined by the need for reintervention (such as balloon dilation) or re-operation (such as conversion from a sleeve gastrectomy to a Roux-en-Y gastric bypass) for stricture, and

- Injury to surrounding tissue as defined by intraoperative repair of an adjacent organ (small or large bowel repair or resection due to injury, repair of the diaphragm, repair 

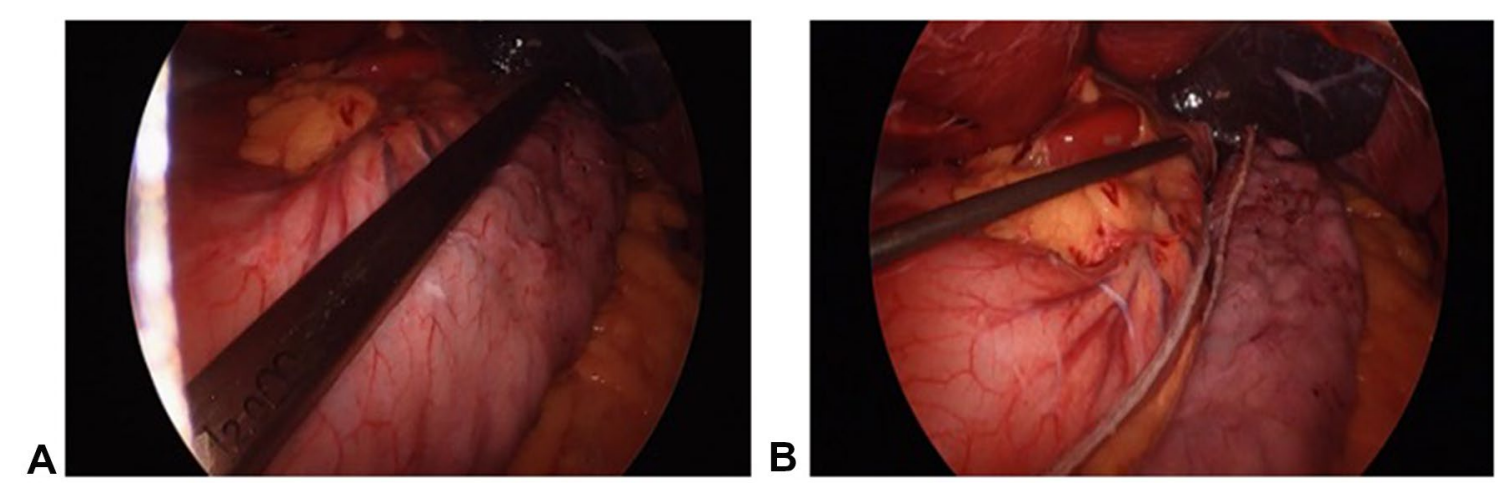

Fig. 2 Intraoperative view of Titan SGS Stapler configuration with stomach in gastric resection $\mathbf{A}$ before and $\mathbf{B}$ after firing

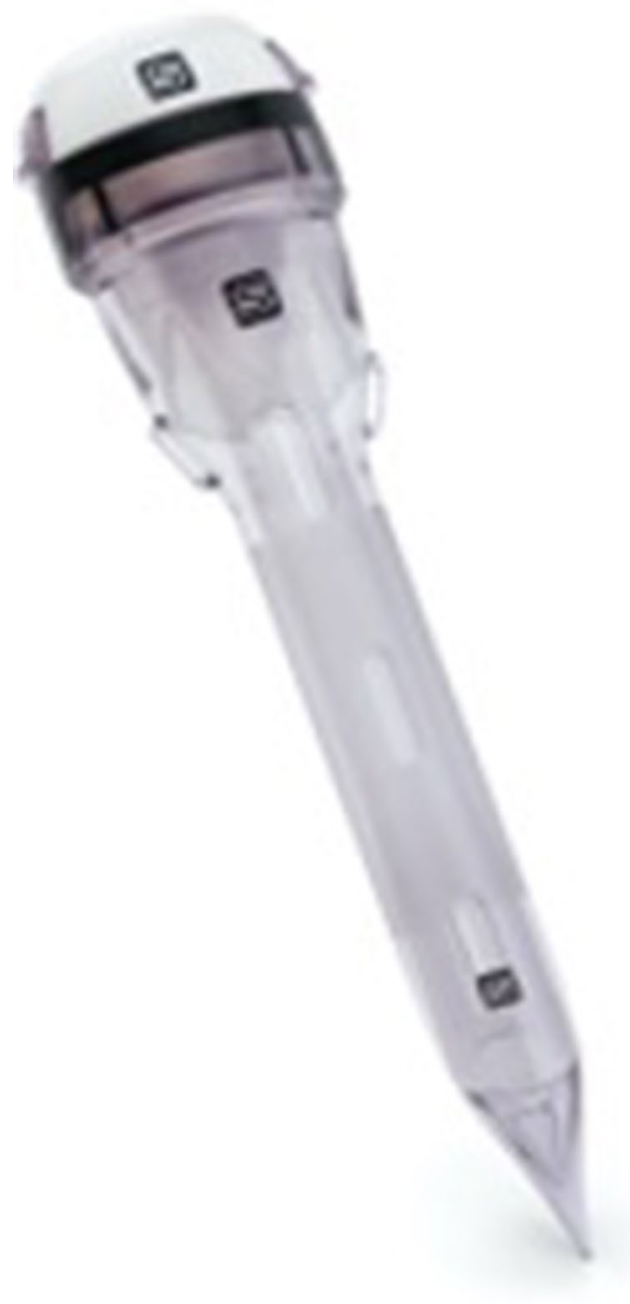

Fig. $319 \mathrm{~mm}$ Standard Trocar for use with Titan SGS stapler of the liver, repair or removal of the spleen) need for re-operation for missed injury directly due to the stapler

\section{Perioperative care and follow-up}

Demographic data were collected pre-operatively including age, sex, race, height, weight, and indication for operation. Intraoperatively, longitudinal gastric resection was undertaken as the surgeon would perform the procedure in standard form. After mobilization of the stomach, the Standard Trocar was placed (Fig. 3), and the Titan SGS stapler (Fig. 1) was introduced. A timeout was taken prior to firing to ensure proper placement of the stapler along the gastric curve (Fig. 2A). After firing the stapler, the resultant anatomy was assessed for bleeding immediately, and any interventions for hemostasis noted (Fig. 2B). An air bubble leak test was then performed. The resected portion of stomach was removed from the Standard Trocar site and analyzed for tissue thickness. Trocar sites were closed according to routine surgeon practice. On post-operative day 1, an upper GI study was obtained per local protocol (as demonstrated in Fig. 4). Both surgeon and radiologist assessment were collected.

The index hospital stay was recorded in terms of length of stay and any adverse events. Readmissions, if any, were recorded and adverse events noted. A 6-week follow-up period was included to determine the occurrence of any possible subacute device-related adverse events.

\section{Statistical methods}

Statistical analysis was performed using SAS version 9.4 (SAS Institute Inc., Cary, NC 27513, USA). Continuous variables were summarized using the following standard descriptive summary statistics: number of observations, arithmetic mean, standard deviation, minimum, lower quartile, median, upper quartile, and maximum. Categorial data were described using absolute and relative frequencies. 


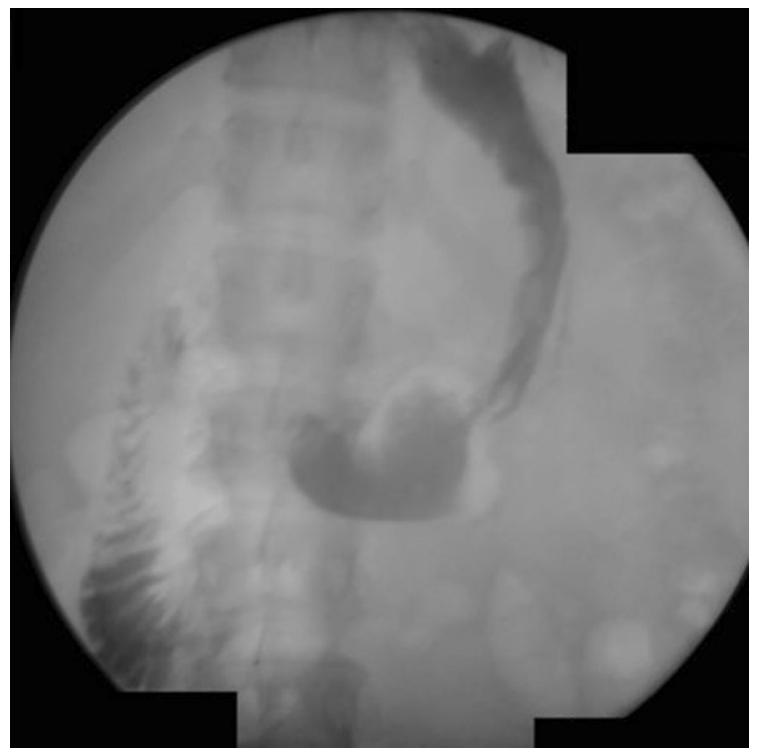

Fig. 4 Example of Upper GI study

Confidence intervals were to be understood as two-sided confidence intervals.

\section{Results}

Sixty-two participants were enrolled between July 2, 2020 and November 3, 2020, and a 6-week follow-up visit to assess any additional adverse events was conducted for all evaluable participants. All participants completed study follow-up. 61/62 patients met all inclusion and exclusion criteria. One participant was mistakenly enrolled after failure to pre-operatively recognize the exclusion criterion of Stage III CKD. This participant was excluded from the perprotocol analysis. A summary of the patient demographic data is included as Table 1, categorized by surgical procedure. Study inclusion criteria specified patients requiring longitudinal gastric resection including LSG, laparoscopic gastric wedge resection, and laparoscopic duodenal switch/ loop duodenal switch.

Intraoperatively, interventions were used to manage minor bleeding along the staple line. As these maneuvers are common in LSG practice, they were not considered adverse events. Intraoperative interventions utilized are included in Table 2. The mean duration of the procedure was $85.6 \mathrm{~min}$ with a minimum of $32 \mathrm{~min}$ and a maximum of $139 \mathrm{~min}$. Study endpoint evaluations for patients enrolled are summarized in Table 3. Surgeon feedback was overwhelmingly positive, as well as feedback from enrolled patients at follow-up.

A study stopping rule was included in order to pause the study in the event that greater than two DRAEs occurred during this study. This threshold was not met over the duration of the study period. Adverse events were collected through 6-week follow-up for all participants. There were no UADEs reported during the study. A review of the adverse events by classification is summarized by type in Table 4. These adverse events were found within the study population but are also common among patients undergoing LSG.

The one DRAE which occurred during the study was a staple line bleed. The patient experienced post-operative tachycardia without hypotension and did not require administration of blood products but underwent takeback operation for abdominal washout per surgeon discretion on postoperative day 1 . No specific bleeding vessel identified at the time of re-operation and the patient was discharged to home on post-operative day 2, prolonging the hospital stay by one day.

There were two other bleeding complications in the study that were determined to not be due to the staple line. One was a post-operative hemorrhage requiring transfusion from a peri-splenic vessel partially torn and inadequately addressed during dissection. This attribution was based on procedural video review by operating surgeon and study PI. The other bleeding event was a delayed bleed from the duodenal dissection bed observed on diagnostic laparoscopy at post-operative day 7. This attribution was based on CT scan and takeback video reviewed by operating surgeon and study PI. Additionally, this patient had been discharged on prophylactic rivaroxaban (2.5 mg BID), which may have been a contributing factor to the delayed bleeding event.

\section{Discussion}

This multicenter study aimed to determine the safety and efficacy of a newly available linear gastric stapler, the Titan SGS, for use in longitudinal gastric resection. Laparoscopic sleeve gastrectomy has become the most common bariatric procedure performed in the United States, and alongside the loop duodenal switch, which was also performed on this patient population, needs standardization for optimization of outcomes. This stapler was created to simplify and streamline the procedure, which could minimize surgeon variability, lead to shorter operative times, and improve standardization of the sleeve structure.

The Titan SGS stapler evolved from the Standard Clamp (Standard Bariatrics, Cincinnati, $\mathrm{OH}$ ), a $25 \mathrm{~cm}$ disposable atraumatic tissue clamp designed to guide stapler placement along the gastric curvature [13]. This clamp, which has been used in over 10,000 laparoscopic sleeve gastrectomies, allows for fixation of the full gastric staple line before and during staple fires for a clear visualization of the linear cut in order to avoid zig-zag or spiraling of the staple line. All four participating surgeons had previous experience with use of the Standard Clamp in longitudinal sleeve gastrectomies. 
Table 1 Patient demographic data

Laparoscopic sleeve gastrectomy $(N=52)$

Age (years)

Mean (SD)

$\operatorname{Sex}[n(\%)]$

Female

Male

Height $(\mathrm{cm})$

Mean (SD)

Weight $(\mathrm{kg})$

Mean (SD)

BMI $\left(\mathrm{kg} / \mathrm{m}^{2}\right)$

Mean (SD)

Race $[n(\%)]$

Black

White

Ethnicity [ $n(\%)]$

Hispanic or Latino

Non-Hispanic, Non- Latino

Unknown/Not reported

ASA class $[n(\%)]$

Mild Systemic Disease

Severe systemic disease

Severe systemic disease/Constant threat to life

Diabetes $[n(\%)]$

No

Yes: Insulin

Yes: Non-Insulin

Functional status $[n(\%)]$

Independent

Table 2 Staple line

interventions

Laparoscopic duodenal switch/loop duodenal switch $(N=9)$

\begin{tabular}{|c|c|c|}
\hline 40.1 (9.99) & $44.0(10.27)$ & 40.7 (10.04) \\
\hline $41(79)$ & $8(89)$ & $49(80)$ \\
\hline $11(21)$ & $1(11)$ & $12(20)$ \\
\hline $167.5(9.87)$ & $166.6(8.37)$ & $176.4(9.60)$ \\
\hline $129.1(25.97)$ & $131.7(29.18)$ & $129.5(26.23)$ \\
\hline $45.9(7.45)$ & $47.6(10.89)$ & $46.2(7.96)$ \\
\hline $11(21)$ & $2(22)$ & $12(21)$ \\
\hline $41(79)$ & $7(78)$ & $48(79)$ \\
\hline $2(4)$ & $1(11)$ & $13(21)$ \\
\hline $37(71)$ & $8(89)$ & $45(74)$ \\
\hline $13(25)$ & $0(0)$ & $13(21)$ \\
\hline $12(23)$ & $0(0)$ & $12(20)$ \\
\hline $38(73)$ & $5(56)$ & $43(70)$ \\
\hline $2(4)$ & $4(44)$ & $6(10)$ \\
\hline $39(75)$ & $4(44)$ & $43(70)$ \\
\hline $5(10)$ & $2(22)$ & $7(11)$ \\
\hline $8(15)$ & $3(33)$ & $11(18)$ \\
\hline $52(100)$ & $9(100)$ & $61(100)$ \\
\hline
\end{tabular}

\begin{tabular}{ll}
\hline & $\begin{array}{l}\text { Per-protocol } \\
\text { population } N \\
(\%)\end{array}$ \\
\hline $\begin{array}{l}\text { Any intervention to manage minor bleeding along the staple line }[n(\%)] \\
\geq 1\end{array}$ & $39 / 61(64 \%)$ \\
None & $22 / 61(36 \%)$ \\
Clips used to manage minor bleeding along staple line $[n(\%)]$ & \\
No & $47 / 61(77 \%)$ \\
Yes & $14 / 61(23 \%)$ \\
Cautery used to manage minor bleeding along staple line $[n(\%)]$ & \\
No & $41 / 61(67 \%)$ \\
Yes & $20 / 61(33 \%)$ \\
\hline
\end{tabular}

The Titan stapler is an advancement from this, removing the need for repeated or overlapping short staple fires. With the ability to plan and place a single line of staples in one fire, surgeon and device variation are removed, to maximize consistency in sleeve gastrectomy outcomes.
Study endpoints were met with no deviation from expected outcomes. (Need to expand on this some.) Adverse events by classification were all anticipated relating to the procedure and in line with what is currently ordinary standard post-operative events. Most adverse events were 
Table 3 Summary of study endpoint outcomes-Per Protocol population

Primary endpoint

Study outcome-per-protocol population $N(\%)$

Rate of Device-Related Adverse Events (DRAEs)

$1 / 61(1.6 \%)$

Secondary endpoints

Rate of Unaticipated Device-Related Adverse Effects (UADEs) by Seriousness

Study outcome-per-protocol population $N(\%)$ $0 / 61$

Rate of Anticipated, non-serious events

Rate of Serious Adverse Events (SAEs) by relatedness

59 AEs in 34 participants

$1 / 61(1.6 \%)$

Usability of the Titan SGS to resect the stomach as determined by intraoperative assessment by

$61 / 61(100 \%)$ rated acceptable

the Surgeon

Table 4 Summary of AEs by type-Per-protocol population

\begin{tabular}{|c|c|c|c|c|}
\hline $\mathrm{AE}$ & Count & Severe Count & SAE & Ongoing \\
\hline \multicolumn{5}{|l|}{ General } \\
\hline Pain (not specific to incisions) & 6 & & & \\
\hline Fatigue/ lethargy & 4 & & & 2 \\
\hline \multicolumn{5}{|l|}{ FEN/GI } \\
\hline Nausea/ vomiting & 7 & & & 1 \\
\hline Constipation & 15 & & & 3 \\
\hline Stomach virus & 1 & & & \\
\hline H. Pylori & 1 & & & \\
\hline Hypokalemia & 1 & & & \\
\hline Hunger & 1 & & & 1 \\
\hline Dehydration & 2 & & & 2 \\
\hline Dysphagia & 2 & & & \\
\hline Reflux/heartburn/indigestion & 3 & & & 2 \\
\hline \multicolumn{5}{|l|}{ Cardiovascular } \\
\hline Orthostatic hypotension & 1 & & & \\
\hline \multicolumn{5}{|l|}{ Pulmonary } \\
\hline Negative eval for PE and pneumonia & 1 & & & \\
\hline \multicolumn{5}{|l|}{ Hematological } \\
\hline Staple line bleeding & 1 & 1 & 1 & \\
\hline Post-op Hemorrhage requiring transfusion & 1 & 1 & 1 & \\
\hline Post-op hematoma & 1 & & 1 & \\
\hline \multicolumn{5}{|l|}{ Integumentary } \\
\hline Wound erythema or itching & 2 & & & \\
\hline Incisional pain & 2 & & & \\
\hline Rash & 2 & & & \\
\hline Wound seepage or hematoma & 3 & & & 1 \\
\hline \multicolumn{5}{|l|}{ Infectious } \\
\hline COVID-19 & 3 & & 1 & \\
\hline \multicolumn{5}{|l|}{ Gynecologic } \\
\hline Yeast infection & 1 & & & \\
\hline Bacterial vaginosis & 1 & & & 1 \\
\hline \multicolumn{5}{|l|}{ Urologic } \\
\hline Kidney stones & 1 & & & \\
\hline Hematuria & 1 & & & \\
\hline Total & 64 & 2 & 4 & 13 \\
\hline
\end{tabular}


classified as mild, with full recovery and no ongoing sequelae, and the majority of events were related to nausea and constipation, both well-known post-operative effects of bariatric surgery. The rate of the use of any intraoperative bleeding interventions was comparable with findings in the literature for currently available staplers (58\% use with Ethicon Echelon GST vs 64\% use with Titan SGS.) [14] Surgeon feedback was positive, in both ratings of the Titan SGS stapler performance, and of surgical outcomes in gastric pouch formation. Additionally, no leaks were detected on upper GI study routinely performed on all participants.

The one device-related adverse outcome of early postoperative bleed requiring re-operation was an anticipated adverse event expected with this procedure and use of staplers in general. Post-operative hemorrhage is an unfortunate and rare complication that is discussed with patients as a risk of any major invasive procedure. Overall incidence of postoperative bleeding in bariatric surgery is reported to be $3 \%$ [15], with half of this due to hemorrhage at the staple line [16]. This patient represents $1.6 \%$ of our study population, comparable to the current benchmarks in bariatric surgery. This patient's complication was identified rapidly after a syncopal event with tachycardia on the patient care floor post-operatively. The patient was intervened on acutely and intraoperatively there was no actively bleeding vessel, cut momentum, or avulsed tissue identified. The patient recovered well, was released to home on post-operative day 2 , with no sequelae of the event. Taking into account the three bleeding events-one device related and two not device related - the overall bleeding event rate for this study was $4.9 \%(3 / 61)$. Although this rate is above the published $3 \%$, the staple line specific bleeding rate was $1.6 \%$, which is comparable to the published $1.5 \%$ benchmark in laparoscopic sleeve gastrectomy.

A limitation of our study is the small sample size of both patients and participating surgeons. Given the overall population of 62 patients and an expected clinical leak rate of $1 \%$, there may not have been enough patients enrolled to have demonstrated a clinically appreciable leak rate, as well as other very low incidence adverse effects. Future studies will be appropriately powered for low incidence outcomes, including staple line bleeding and staple line leak rates.

Previous studies have shown that optimal stapler selection during LSG is of utmost importance [17]. Regarding staple height, taller staples with under compression of gastric tissue can lead to decreased integrity of the staple line, predisposing to leaks or bleeding [18]. However, over compression of the staple line has been historically thought to cause ischemia and also lead to complications [19]. More recent data, however, supports increased compression leading to improved outcomes, with increased hemostasis, decreased incidence of leak [20], and potentially decreased stricture rates [21]. The Titan SGS stapler operates using a graduated staple height formation optimized for gastric tissue [22]. This removes surgeon variation or inaccuracy from the choice of staple height, and outcomes were shown to be consistent with current expected outcomes. The proven safety and efficacy of this stapler may lead to more consistent operative technique and outcomes in bariatric surgery.

\section{Conclusion}

The Titan SGS stapler was both safe and effective in sleeve gastrectomy pouch creation.

\section{Declarations}

Disclosures Dr. Jon Thompson is the founder and CMO of Standard Bariatrics, Inc. Dr. Salyer, Dr. Hoffman, Dr. Burstein, Dr. Enochs, Dr. Watkins, Dr. Kuethe, and Dr. Goodman do not have conflicts of interest or financial ties to disclose. The study was funded by Standard Bariatrics, Inc. via study-specific independent budgeting and contracts with the University of Cincinnati, SUNY Buffalo and WakeMed.

\section{References}

1. Hales CM, Carroll MD, Fryar CD, Ogden CL (2020) Prevalence of obesity and severe obesity among adults: United States, 20172018. NCHS Data Brief 360:1-8

2. English WJ, DeMaria EJ, Hutter MM, Kothari SN, Mattar SG, Brethauer SA et al (2020) American Society for Metabolic and Bariatric Surgery 2018 estimate of metabolic and bariatric procedures performed in the United States. Surg Obes Relat Dis 16(4):457-463. https://doi.org/10.1016/j.soard.2019.12.022

3. Musella M, Milone M, Gaudioso D, Bianco P, Palumbo R, Galloro G, et al (2014) A decade of bariatric surgery What have we learned? Outcome in 520 patients from a single institution. Int $\mathbf{J}$ Surg. 12 (1):183-8. doi: https://doi.org/10.1016/j.ijsu.2014.05.012

4. Wang FG, Yu ZP, Yan WM, Yan M, Song MM (2017) Comparison of safety and effectiveness between laparoscopic mini-gastric bypass and laparoscopic sleeve gastrectomy: a meta-analysis and systematic review. Medicine (Baltimore) 96(50):e8924. https:// doi.org/10.1097/MD.0000000000008924

5. Shoar S, Saber AA (2017) Long-term and midterm outcomes of laparoscopic sleeve gastrectomy versus Roux-en-Y gastric bypass: a systematic review and meta-analysis of comparative studies. Surg Obes Relat Dis 13(2):170-180. https://doi.org/10.1016/j. soard.2016.08.011

6. Juodeikis Ž, Brimas G (2017) Long-term results after sleeve gastrectomy: a systematic review. Surg Obes Relat Dis 13(4):693699. https://doi.org/10.1016/j.soard.2016.10.006

7. Dhar VK, Hanseman DJ, Watkins BM, Paquette IM, Shah SA, Thompson JR (2018) What matters after sleeve gastrectomy: patient characteristics or surgical technique? Surgery 163(3):571577. https://doi.org/10.1016/j.surg.2017.09.052

8. Toro JP, Lin E, Patel AD, Davis SS, Sanni A, Urrego HD et al (2014) Association of radiographic morphology with early gastroesophageal reflux disease and satiety control after sleeve 
gastrectomy. J Am Coll Surg 219(3):430-438. https://doi.org/10. 1016/j.jamcollsurg.2014.02.036

9. Chang PC, Tai CM, Hsin MC, Hung CM, Huang IY, Huang CK (2017) Surgical standardization to prevent gastric stenosis after laparoscopic sleeve gastrectomy: a case series. Surg Obes Relat Dis 13(3):385-390. https://doi.org/10.1016/j.soard.2016.09.014

10. Sun SD, Wu PP, Zhou JF, Wang ZS, Cai TH, Huang LK et al (2020) Comparison of single-incision and conventional laparoscopic sleeve gastrectomy for morbid obesity: a meta-analysis. Obes Surg 30(6):2186-2198. https://doi.org/10.1007/ s11695-020-04437-z

11. Baker RS, Foote J, Kemmeter P, Brady R, Vroegop T, Serveld M (2004) The science of stapling and leaks. Obes Surg 14(10):12901298. https://doi.org/10.1381/0960892042583888

12. Salyer C, Spuzzillo A, Wakefield D, Gomaa D, Thompson J, Goodman M (2020) Assessment of a novel stapler performance for laparoscopic sleeve gastrectomy. Surg Endosc. https://doi.org/ 10.1007/s00464-020-07858-0

13. Ashar BS (2018) FDA Clearance Letter; Regulation Number: 21 CFR 876.1500. In: Administration USFaD (ed)

14. Fegelman E, Knippenberg S, Schwiers M, Stefanidis D, Gersin KS, Scott JD et al (2017) Evaluation of a powered stapler system with gripping surface technology on surgical interventions required during laparoscopic sleeve gastrectomy. J Laparoendosc Adv Surg Tech A 27(5):489-494. https://doi.org/10.1089/lap. 2016.0513

15. Karmali S, Johnson Stoklossa C, Sharma A, Stadnyk J, Christiansen S, Cottreau D et al (2010) Bariatric surgery: a primer. Can Fam Phys 56(9):873-879

16. De Angelis F, Abdelgawad M, Rizzello M, Mattia C, Silecchia G (2017) Perioperative hemorrhagic complications after laparoscopic sleeve gastrectomy: four-year experience of a bariatric center of excellence. Surg Endosc 31(9):3547-3551. https://doi. org/10.1007/s00464-016-5383-y

17. Chekan E, Whelan RL (2014) Surgical stapling device-tissue interactions: what surgeons need to know to improve patient outcomes. Med Devices (Auckl) 7:305-318. https://doi.org/10.2147/ MDER.S67338

18. Ghosh SK, Roy S, Chekan E, Fegelman EJ (2016) A narrative of intraoperative staple line leaks and bleeds during bariatric surgery. Obes Surg 26(7):1601-1606. https://doi.org/10.1007/ s11695-016-2177-1

19. Elariny H, González H, Wang B (2005) Tissue thickness of human stomach measured on excised gastric specimens from obese patients. Surg Technol Int 14:119-124

20. Sakran N, Assalia A, Sternberg A, Kluger Y, Troitsa A, Brauner E et al (2011) Smaller staple height for circular stapled gastrojejunostomy in laparoscopic gastric bypass: early results in 1,074 morbidly obese patients. Obes Surg 21(2):238-243. https://doi. org/10.1007/s11695-010-0308-7

21. Hanna K, Seder CW, Chengelis D, McCullough PA, Krause K (2012) Shorter circular staple is height associated with lower anastomotic stricture rate in laparoscopic gastric bypass. Surg Obes Relat Dis 8(2):181-184. https://doi.org/10.1016/j.soard.2011.01. 046

22. Yeo E, Thompson J, Hanseman D, Dunki-Jacobs A, Thompson B, Goodman $\mathrm{M}$ et al (2020) Increased staple loading pressures and reduced staple heights in laparoscopic sleeve gastrectomy reduce intraoperative bleeding. Surgery. https://doi.org/10.1016/j.surg. 2020.10.045

Publisher's Note Springer Nature remains neutral with regard to jurisdictional claims in published maps and institutional affiliations. 